\title{
Global Exponential Stability for DCNNs with Impulses on Time Scales
}

\author{
Yongkun Li and Yuanhong Zhi \\ Department of Mathematics, Yunnan University, Kunming, Yunnan 650091, China \\ Correspondence should be addressed to Yongkun Li; yklie@ynu.edu.cn
}

Received 25 September 2013; Accepted 17 December 2013; Published 2 January 2014

Academic Editor: Lu Zhen

Copyright (C) 2014 Y. Li and Y. Zhi. This is an open access article distributed under the Creative Commons Attribution License, which permits unrestricted use, distribution, and reproduction in any medium, provided the original work is properly cited.

\begin{abstract}
A class of delayed cellular neural networks (DCNNs) with impulses on time scales is considered. By using the topological degree theory, and the time scale calculus theory some sufficient conditions are derived to ensure the existence, uniqueness, and global exponential stability of equilibria for this class of neural networks. Finally, a numerical example illustrates the feasibility of our results and also shows that the continuous-time neural network and the discrete-time analogue have the same dynamical behaviors. The results of this paper are completely new and complementary to the previously known results.
\end{abstract}

\section{Introduction}

Chua and Yang [1] proposed a novel class of informationprocessing systems called cellular neural networks (CNNs) in 1988. The CNNs can be applied in signal processing and can also be used to solve some image processing and pattern recognition problems [2]. Since time delays are unavoidable due to finite switching speeds of the amplifiers, delayed cellular neural networks (DCNNs) have been widely studied and successfully applied to pattern recognition, associative memories, and signal processing and optimization, especially in image processing. The dynamic behavior of the networks plays an important role in such applications [3-8]. Therefore, there are many works on the stability of equilibrium point of delayed cellular neural networks (DCNNs) [5-13].

Most neural networks can be classified into two types: continuous or discrete. However, many real-world systems and natural processes cannot be categorized into one of them. They display characteristics of both continuous and discrete styles. For instance, some biological neural networks in biology, bursting rhythm models in pathology, and optimal control models in economics are characterized by abrupt changes of state. These are the familiar impulsive phenomena. Other examples can also be found in information science, electronics, automatic control systems, computer networking, artificial intelligence, robotics, telecommunications, and so forth. Such a kind of phenomena, in which sudden and sharp changes often occur in a continuous process, cannot be well described by pure continuous or pure discrete models. Therefore, it is important and, in effect, necessary to study a new type of neural networks-impulsive neural networks-as an appropriate description of these phenomena of abrupt qualitative dynamical changes of essentially continuous systems. The fundamental theory of impulsive differential equations has been developed in [14]. Since delays and impulses can affect the dynamical behaviors of the system, it is necessary to investigate both delay and impulsive effects on the stability of neural networks. For more details, one can refer to $[10,13,15-23]$.

The theory of time scale was initiated by Hilger in 1988, which has recently received a lot of attention [2426]. The field of dynamic equations on time scale contains links and extends the classical theory of differential and difference equations. It is well known that both continuous and discrete systems are very important in implementation and applications (see [27-30]). But it is troublesome to study the stability for continuous and discrete systems, respectively. 
Therefore, it is significant to study that on time scales which can unify the continuous and discrete situations [21, 31-40].

Motivated by above, in this paper, we are concerned with the following impulsive DCNN on time scales:

$$
\begin{gathered}
x_{i}^{\Delta}(t)=-a_{i} x_{i}(t)+\sum_{j=1}^{n} b_{i j} f_{j}\left(x_{j}(t)\right) \\
+\sum_{j=1}^{n} c_{i j} f_{j}\left(x_{j}\left(\zeta_{i j}\left(t, x_{j}(t)\right)\right)\right)+I_{i}, \\
t \in \mathbb{T}_{0}^{+}, t \neq t_{k}, \\
x_{i}\left(t_{k}^{+}\right)=x_{i}\left(t_{k}^{-}\right)+P_{i}\left(x_{i}\left(t_{k}^{-}\right)\right), \\
k=1,2, \ldots, i=1,2, \ldots, n,
\end{gathered}
$$

where $n$ corresponds to the numbers of units in a neural network; $x_{i}(t)$ corresponds to the state of the $i$ th unit at time $t$; $f_{j}\left(x_{j}(t)\right)$ denotes the output of the $j$ th unit at time $t . \mathbb{T}_{0}^{+}$is the $\mathbb{T}$-interval $\{t \in \mathbb{T}, t \geq 0\}$, and $\mathbb{T}$ denotes a time scale, which is an arbitrary nonempty closed subset of the real number $\mathbb{R}$ and with bounded graininess $\mu$. For the simplicity, we assume that $0 \in \mathbb{T}$ and $\mathbb{T}$ is unbounded above; that is, sup $\mathbb{T}=+\infty$. Further, $b_{i j}, c_{i j}, a_{i}$, and $I_{i}$ are constants. $b_{i j}, c_{i j}$ denote the strength of the $j$ th unit at time $t$ and $\zeta_{i j}\left(t, x_{j}(t)\right)$, respectively. $I_{i}$ denotes the external bias on the $i$ th unit and $a_{i}$ represents the rate with which the $i$ th unit will reset its potential to the resting state in isolation when disconnected from the network and external inputs. $t_{k}, k=1,2, \ldots$ are the moments of impulsive perturbations and satisfy $0=t_{0}<t_{1}<t_{2}<\cdots$ and $\lim _{k \rightarrow \infty}=\infty, \mu\left(t_{k}\right)=0$ (see Definition 3). $P_{i}\left(x_{i}\left(t_{k}\right)\right.$ ) represents the abrupt change of the state $x_{i}(t)$ at the impulsive moment $t_{k}$. To the best of our knowledge, this is first paper to study DCNNs with impulses on time scales.

Throughout this paper, we assume that $x_{i}\left(t_{k}\right) \equiv x_{i}\left(t_{k}^{-}\right)$and

(H1) functions $\zeta_{i j}$ satisfy $\zeta_{i j}: \mathbb{T} \times \mathbb{R} \rightarrow \mathbb{T}$ for all $t \in \mathbb{T}$, $i, j=1,2, \ldots, n$;

$(\mathrm{H} 2) f_{j} \in C(\mathbb{R}, \mathbb{R})(i=1,2, \ldots, n)$ and there exists a positive number $F_{i}$ such that $\left|f_{i}(x)-f_{i}(y)\right| \leq F_{i}|x-y|$ for all $x, y \in \mathbb{R}, i=1,2, \ldots, n$.

Remark 1. The neural network (1) is a system of differential equations with state-dependent deviating arguments and from ( $\mathrm{H} 1)$, one can see that deviating arguments in (1) may be delayed type, advanced type, or mixed type.

Our main purpose of this paper is to study the existence and global exponential stability of the equilibria of (1) by using the topological degree theory and the time scale calculus theory. The results of this paper are completely new and complementary to the previously known results.

The organization of this paper is as follows. In the next section, some notations, definitions, and lemmas are presented. Section 3 addresses the existence and uniqueness of equilibria of system (1) by using the method of topological degree theory. In Section 4, we give the criteria of global exponential stability of the equilibrium point of system (1). In Section 5, an example is also provided to illustrate the effectiveness of the main results in Sections 3 and 4.

\section{Notations and Preliminaries}

In this section, we will first recall some basic definitions and lemmas which will be useful for the proof of our main results.

Definition 2 (see $[33,34])$. A time scale $\mathbb{T}$ is arbitrary nonempty closed subset of the real set $\mathbb{R}$ with the topology and ordering inherited from $\mathbb{R}$.

Definition 3 (see $[33,34]$ ). On any time scale $\mathbb{T}$, we define the forward and backward jump operators by

$$
\sigma(t):=\inf \{s \in \mathbb{T}: s>t\}, \quad \rho(t):=\sup \{s \in \mathbb{T}: s<t\} .
$$

A point $t$ is said to be left-dense if $t>\inf \mathbb{T}$ and $\rho(t)=t$, rightdense if $t<\sup \mathbb{T}$ and $\sigma(t)=t$, left-scattered if $\rho(t)<t$, and right-scattered if $\sigma(t)>t$. The graininess function $\mu$ for a time scale $\mathbb{T}$ is defined by $\mu(t):=\sigma(t)-t$. If $\mathbb{T}$ has a left-scattered maximum $m$, then we defined $\mathbb{T}^{k}$ to be $\mathbb{T} \backslash\{m\}$. Otherwise, $\mathbb{T}^{k}=\mathbb{T}$.

Definition 4 (see $[33,34]$ ). For a function $f: \mathbb{T} \rightarrow \mathbb{R}$ (the range $\mathbb{R}$ of $f$ may be actually replaced by Banach space), the (delta) derivative is defined by

$$
f^{\Delta}=\frac{f(\sigma(t))-f(t)}{\sigma(t)-t}
$$

if $f$ is continuous at $t$ and $t$ is right-scattered. If $t$ is not rightscattered, then the derivative is defined by

$$
f^{\Delta}=\lim _{s \rightarrow t, s \in \mathbb{T}} \frac{f(\sigma(t))-f(s)}{\sigma(t)-s}=\lim _{s \rightarrow t, s \in \mathbb{T}} \frac{f(t)-f(s)}{t-s},
$$

provided this limit exists.

Lemma 5 (see $[33,34])$. If $f$, $g$ are differential at $t \in \mathbb{T}$, one has

(1) $f(\sigma(t))=f(t)+\mu(t) f^{\Delta}(t)$;

(2) $(f g)^{\Delta}(t)=f^{\Delta}(t) g(t)+f(\sigma(t)) g^{\Delta}(t)$.

Definition 6 (see $[33,34]$ ). A function $F: \mathbb{T}^{k} \rightarrow \mathbb{R}$ is called a delta-antiderivative of $f: \mathbb{T} \rightarrow \mathbb{R}$ provided $F^{\Delta}=f$ holds for all $t \in \mathbb{T}^{k}$. In this case, we define the integral of $f$ by

$$
\int_{a}^{t} f(s) \Delta s=F(t)-F(a) \quad \text { for } t \in \mathbb{T},
$$

and we have the following formula:

$$
\int_{t}^{\sigma(t)} f(s) \Delta s=\mu(t) f(t) \quad \text { for } t \in \mathbb{T}^{k} .
$$

Definition 7 (see $[33,34]$ ). A function $f: \mathbb{T} \rightarrow \mathbb{R}$ is called right-dense continuous (rd-continuous) provided it is continuous at right-dense points of $\mathbb{T}$ and the left-sided limit exists (finite) at left-dense point of $\mathbb{T}$. The set of all right-dense continuous functions on $\mathbb{T}$ is defined by $C_{\mathrm{rd}}=C_{\mathrm{rd}}(\mathbb{T})=$ $C_{\text {rd }}(\mathbb{T}, \mathbb{R})$. If $f$ is continuous at each right-dense point and each left-dense point, then $f$ is said to be continuous function on $\mathbb{T}$. We define $C(J, \mathbb{R})=\{f(t)$ is continuous on $J\}$. 
Lemma 8 (see $[33,34]$ ). If $a, b \in \mathbb{T}, \alpha, \beta \in \mathbb{R}$ and $f, g \in$ $C(\mathbb{T}, \mathbb{R})$, then one has

(1) $\int_{a}^{b}[\alpha f(t)+\beta g(t)] \Delta t=\alpha \int_{a}^{b} f(t) \Delta t+\beta \int_{a}^{b} g(t) \Delta t ;$

(2) if $f(t) \geq 0$ for all $a \leq t<b$, then $\int_{a}^{b} f(t) \Delta t \geq 0$;

(3) if $|f(t)| \leq g(t)$ on $[a, b):=\{t \in \mathbb{T}: a \leq t<b\}$, then $\left|\int_{a}^{b} f(t) \Delta t\right| \leq \int_{a}^{b} g(t) \Delta t$.

Definition 9 (see $[33,34]$ ). A function $p: \mathbb{T} \rightarrow \mathbb{R}$ is called regressive if $1+\mu(t) p(t) \neq 0$ for all $t \in \mathbb{T}$. If $p$ is regressive function, then the generalized exponential function $e_{p}$ is defined by

$$
e_{p}(t, s)=\exp \left\{\int_{s}^{t} \xi_{\mu(\tau)}(r(\tau)) \Delta \tau\right\}, \quad \text { for } s, t \in \mathbb{T},
$$

with the cylinder transformation

$$
\xi_{h}(z)= \begin{cases}\frac{\log (1+h z)}{h}, & \text { if } h \neq 0, \\ z, & \text { if } h=0 .\end{cases}
$$

Let $p, q: \mathbb{T} \rightarrow \mathbb{R}$ be two regressive functions; we define

$$
\begin{gathered}
p \oplus q:=p+q+\mu p q, \quad \ominus p:=-\frac{p}{1+\mu p}, \\
p \ominus q:=p \oplus(\ominus q) .
\end{gathered}
$$

Then, the generalized exponential function has the following properties.

Lemma 10 (see $[33,34])$. Assume that $p, q: \mathbb{T} \rightarrow \mathbb{R}$ are two regressive functions; then

(1) $e_{0}(t, s) \equiv 1$ and $e_{p}(t, t) \equiv 1$;

(2) $e_{p}(\sigma(t), s)=(1+\mu(t) p(t)) e_{p}(t, s)$;

(3) $e_{p}(t, \sigma(s))=e_{p}(t, s) /(1+\mu(s) p(s))$;

(4) $1 / e_{p}(t, s)=e_{\ominus p}(t, s)$;

(5) $e_{p}(t, s)=1 / e_{p}(s, t)=e_{\ominus p}(s, t)$;

(6) $e_{p}(t, s) e_{p}(s, r)=e_{p}(t, r)$;

(7) $e_{p}(t, s) e_{q}(t, s)=e_{p \oplus q}(t, s)$;

(8) $e_{p}(t, s) / e_{q}(t, s)=e_{p \ominus q}(t, s)$.

Definition 11. A point $x^{*}=\left(x_{1}^{*}, x_{2}^{*}, \ldots, x_{n}^{*}\right)^{T} \in \mathbb{R}^{n}$ is called an equilibrium point of model (1) if $x(t)=x^{*}$ is a solution of (1).

Throughout this paper, we always assume that the impulsive jump vector $P$ satisfies

$$
P\left(x^{*}\right)=\left(P_{1}\left(x_{1}^{*}\right), P_{2}\left(x_{2}^{*}\right), \ldots, P_{n}\left(x_{n}^{*}\right)\right)^{T}=0 .
$$

That is, if $x^{*}$ is an equilibrium point of the following nonimpulsive system:

$$
\begin{array}{r}
x_{i}^{\Delta}(t)=-a_{i} x_{i}(t)+\sum_{j=1}^{n} b_{i j} f_{j}\left(x_{j}(t)\right) \\
+\sum_{j=1}^{n} c_{i j} f_{j}\left(x_{j}\left(\zeta_{i j}\left(t, x_{j}(t)\right)\right)\right)+I_{i}, \\
i=1,2, \ldots, n,
\end{array}
$$

then it is also the equilibrium point of impulsive system (1).

Definition 12 (see [41]). A real matrix $D=\left(d_{i j}\right)_{n \times n}$ is said to be a nonsingular $M$-matrix if $d_{i j} \leq 0(i, j=1,2, \ldots, n, i \neq j)$, and all successive principal minors of $D$ are positive.

Lemma 13 (see [41]). Let $D=\left(d_{i j}\right)_{n \times n}$ with $d_{i j} \leq 0(i, j=$ $1,2, \ldots, n, i \neq j)$; then $D$ is a nonsingular $M$-matrix if and only if the diagonal elements of $D$ are all positive and there exists a positive vectord such that $D d>0$ or $D^{T} d>0$.

\section{Existence and Uniqueness of Equilibrium Point}

In this section, we will discuss the existence and uniqueness of equilibria of the DCNN with impulses on time scales and give their proofs.

Theorem 14. Under assumptions (H1) and (H2), if the following condition is satisfied

(H) $a_{i}-\sum_{j=1}^{n} F_{j}\left(\left|b_{i j}\right|+\left|c_{i j}\right|\right)>0, i=1,2, \ldots, n$,

then there is exactly one equilibrium point of model (1).

Remark 15. From Lemma 13, we can easily prove that $(\mathrm{H})$ holds implying that the following condition is true:

(H0) there exists a vector $\xi=\left(\xi_{1}, \xi_{2}, \ldots, \xi_{n}\right)^{T}>0$ such that

$$
a_{i} \xi_{i}-F_{i} \sum_{j=1}^{n}\left(\left|b_{j i}\right|+\left|c_{j i}\right|\right) \xi_{j}>0, \quad i=1,2, \ldots, n .
$$

For convenience, we set $A=\operatorname{diag}\left(a_{1}, a_{2}, \ldots, a_{n}\right), B=$ $\left(\left|b_{i j}\right|\right)_{n \times n}, C=\left(\left|c_{i j}\right|\right)_{n \times n}$, and $F=\operatorname{diag}\left(F_{1}, F_{2}, \ldots, F_{n}\right)$. Let $\eta=(\underbrace{1,1, \ldots, 1}_{n})^{T}, D=A-(B+C) F$. From assumption $(\mathrm{H})$, we have

$$
\begin{aligned}
D \eta & =\left[\begin{array}{c}
a_{1}-\sum_{j=1}^{n} F_{j}\left(\left|b_{1 j}\right|+\left|c_{1 j}\right|\right) \\
a_{2}-\sum_{j=1}^{n} F_{j}\left(\left|b_{2 j}\right|+\left|c_{2 j}\right|\right) \\
\vdots \\
a_{n}-\sum_{j=1}^{n} F_{j}\left(\left|b_{n j}\right|+\left|c_{n j}\right|\right)
\end{array}\right)>0,
\end{aligned}
$$


which implies that $D$ is a nonsingular $M$-matrix. So we know that $D^{T}$ is a nonsingular $M$-matrix. Hence, there exists a vector $\xi=\left(\xi_{1}, \xi_{2}, \ldots, \xi_{n}\right)^{T}>0$ such that

$$
\begin{aligned}
D^{T} \xi= & {\left[A^{T}-F^{T}\left(B^{T}+C^{T}\right)\right] \xi } \\
& =\left(\begin{array}{c}
a_{1} \xi_{1}-F_{1} \sum_{j=1}^{n}\left(\left|b_{j 1}\right|+\left|c_{j 1}\right|\right) \xi_{j} \\
a_{2} \xi_{2}-F_{2} \sum_{j=1}^{n}\left(\left|b_{j 2}\right|+\left|c_{j 2}\right|\right) \xi_{j} \\
\vdots \\
a_{n} \xi_{n}-F_{n} \sum_{j=1}^{n}\left(\left|b_{j n}\right|+\left|c_{j n}\right|\right) \xi_{j}
\end{array}\right)>0 .
\end{aligned}
$$

It follows that (H0) holds.

Now, we prove our theorem.

Proof. Let $x^{*}=\left(x_{1}^{*}, x_{2}^{*}, \ldots, x_{n}^{*}\right)^{T}$ be an equilibrium point of system (1); then, we have

$$
\begin{array}{r}
-a_{i} x_{i}^{*}+\sum_{j=1}^{n} b_{i j} f_{j}\left(x_{j}^{*}\right)+\sum_{j=1}^{n} c_{i j} f_{j}\left(x_{j}^{*}\right)+I_{i}=0, \\
i=1,2, \ldots, n .
\end{array}
$$

We denote $h\left(x_{1}, x_{2}, \ldots, x_{n}\right)=\left(h_{1}, h_{2}, \ldots, h_{n}\right)^{T}$, where

$$
\begin{array}{r}
h_{i}=a_{i} x_{i}-\sum_{j=1}^{n} b_{i j} f_{j}\left(x_{j}\right)-\sum_{j=1}^{n} c_{i j} f_{j}\left(x_{j}\right)-I_{i}, \\
i=1,2, \ldots, n .
\end{array}
$$

Obviously, the equilibrium points of model (1) are solutions of system

$$
h_{i}=0, \quad i=1,2, \ldots, n .
$$

Define the following homotopic mapping:

$$
\begin{aligned}
H\left(x_{1}, x_{2}, \ldots, x_{n}\right)= & \lambda h\left(x_{1}, x_{2}, \ldots, x_{n}\right) \\
& +(1-\lambda)\left(x_{1}, x_{2}, \ldots, x_{n}\right)^{T},
\end{aligned}
$$

where $\lambda \in[0,1]$. Let $H_{k}(k=1,2, \ldots, n)$ denote the $k$ th component of $H\left(x_{1}, x_{2}, \ldots, x_{n}\right)$; then, we can get

$$
\begin{aligned}
\left|H_{i}\right|= & \mid(1-\lambda) x_{i}+\lambda a_{i} x_{i}-\lambda \sum_{j=1}^{n} b_{i j} f_{j}\left(x_{j}\right) \\
& \quad-\lambda \sum_{j=1}^{n} c_{i j} f_{j}\left(x_{j}\right)-\lambda I_{i} \mid \\
\geq & (1-\lambda)\left|x_{i}\right|+\lambda a_{i}\left|x_{i}\right| \\
& -\lambda \sum_{j=1}^{n}\left|b_{i j}\right|\left|f_{j}\left(x_{j}\right)\right|-\lambda \sum_{j=1}^{n}\left|c_{i j}\right|\left|f_{j}\left(x_{j}\right)\right|-\lambda\left|I_{i}\right|
\end{aligned}
$$

$$
\begin{aligned}
& \geq \lambda a_{i}\left|x_{i}\right|-\lambda \sum_{j=1}^{n}\left(\left|b_{i j}\right|+\left|c_{i j}\right|\right)\left|f_{j}\left(x_{j}\right)-f_{j}(0)\right| \\
& \quad-\lambda \sum_{j=1}^{n}\left(\left|b_{i j}\right|+\left|c_{i j}\right|\right)\left|f_{j}(0)\right|-\lambda\left|I_{i}\right| \\
& \geq \lambda a_{i}\left|x_{i}\right|-\lambda \sum_{j=1}^{n} F_{j}\left(\left|b_{i j}\right|+\left|c_{i j}\right|\right)\left|x_{j}\right| \\
& \quad-\lambda \sum_{j=1}^{n}\left(\left|b_{i j}\right|+\left|c_{i j}\right|\right)\left|f_{j}(0)\right|-\lambda\left|I_{i}\right| .
\end{aligned}
$$

It follows that

$$
\begin{aligned}
\sum_{i=1}^{n}\left|H_{i}\right| \geq \sum_{i=1}^{n}[ & \lambda a_{i}\left|x_{i}\right|-\lambda \sum_{j=1}^{n} F_{j}\left(\left|b_{i j}\right|+\left|c_{i j}\right|\right)\left|x_{j}\right| \\
& \left.-\lambda \sum_{j=1}^{n}\left(\left|b_{i j}\right|+\left|c_{i j}\right|\right)\left|f_{j}(0)\right|-\lambda\left|I_{i}\right|\right] \\
\geq & \lambda \sum_{i=1}^{n}\left[a_{i}-\sum_{j=1}^{n} F_{j}\left(\left|b_{i j}\right|+\left|c_{i j}\right|\right)\right]\left|x_{j}\right| \\
& -\lambda \sum_{i=1}^{n}\left[\sum_{j=1}^{n}\left(\left|b_{i j}\right|+\left|c_{i j}\right|\right)\left|f_{j}(0)\right|+\left|I_{i}\right|\right] .
\end{aligned}
$$

Let

$$
\begin{gathered}
\theta=\min _{1 \leq i \leq n}\left\{a_{i}-\sum_{j=1}^{n} F_{j}\left(\left|b_{i j}\right|+\left|c_{i j}\right|\right)\right\}, \\
\gamma=\max _{1 \leq i \leq n}\left\{\left(\left|b_{i j}\right|+\left|c_{i j}\right|\right)\left|f_{j}(0)\right|\right\}, \\
\|x\|_{1}=\sum_{i=1}^{n}\left|x_{i}\right| .
\end{gathered}
$$

From the assumption of the theorem, we can easily see that $\theta>0$. Let

$$
\Omega=\left\{x \mid\|x\|_{1} \leq \frac{n(\gamma+1)}{\theta}\right\} .
$$

Then, for any $x \in \partial \Omega$, we have

$$
\begin{aligned}
\sum_{i=1}^{n}\left|H_{i}\right| & \geq \lambda \theta \sum_{i=1}^{n}\left|x_{i}\right|-\lambda n \gamma \\
& =\lambda \theta \frac{n(\gamma+1)}{\theta}-\lambda n \gamma \\
& >0, \quad \lambda \in(0,1] .
\end{aligned}
$$

As $\lambda=0$, we have

$$
H\left(x_{1}, x_{2}, \ldots, x_{n}\right)=\left(x_{1}, x_{2}, \ldots, x_{n}\right)^{T} \neq 0, \quad x \in \partial \Omega .
$$


Hence, all the above conclusions mean that

$$
H\left(x_{1}, x_{2}, \ldots, x_{n}\right) \neq 0, \quad \text { for any } x \in \partial \Omega, \lambda \in[0,1] \text {. }
$$

From the homotopy invariance theorem, we obtain

$$
\operatorname{deg}(h, \Omega, 0)=\operatorname{deg}(H, \Omega, 0)=\operatorname{deg}(I, \Omega, 0)=1,
$$

where $I$ is the identity operator. By topological degree theory, we can easily know that system (11) has at least one solution in $\Omega$. That means model (1) has at least an equilibrium point.

In order to prove the uniqueness of the equilibrium point, let $x^{*}=\left(x_{1}^{*}, x_{2}^{*}, \ldots, x_{n}^{*}\right)^{T}$ and $y^{*}=\left(y_{1}^{*}, y_{2}^{*}, \ldots, y_{n}^{*}\right)^{T}$ be two equilibrium points of system (1). So, we have

$$
\begin{array}{r}
-a_{i} x_{i}^{*}+\sum_{j=1}^{n} b_{i j} f_{j}\left(x_{j}^{*}\right)+\sum_{j=1}^{n} c_{i j} f_{j}\left(x_{j}^{*}\right)+I_{i}=0, \\
i=1,2, \ldots, n, \\
-a_{i} y_{i}^{*}+\sum_{j=1}^{n} b_{i j} f_{j}\left(y_{j}^{*}\right)+\sum_{j=1}^{n} c_{i j} f_{j}\left(y_{j}^{*}\right)+I_{i}=0, \\
i=1,2, \ldots, n .
\end{array}
$$

Then,

$$
\begin{array}{r}
a_{i}\left(x_{i}^{*}-y_{i}^{*}\right)=\sum_{j=1}^{n}\left(b_{i j}+c_{i j}\right)\left[f_{j}\left(x_{j}^{*}\right)-f_{j}\left(y_{j}^{*}\right)\right], \\
i=1,2, \ldots, n .
\end{array}
$$

By using assumption (H2), we get

$$
\begin{array}{r}
a_{i}\left|x_{i}^{*}-y_{i}^{*}\right| \leq \sum_{j=1}^{n} F_{j}\left(\left|b_{i j}\right|+\left|c_{i j}\right|\right)\left|x_{j}^{*}-y_{j}^{*}\right|, \\
i=1,2, \ldots, n .
\end{array}
$$

It follows that

$$
\begin{array}{r}
a_{i} \xi_{i}\left|x_{i}^{*}-y_{i}^{*}\right| \leq \xi_{i} \sum_{j=1}^{n} F_{j}\left(\left|b_{i j}\right|+\left|c_{i j}\right|\right)\left|x_{j}^{*}-y_{j}^{*}\right|, \\
i=1,2, \ldots, n .
\end{array}
$$

Hence,

$$
\begin{aligned}
\sum_{i=1}^{n} a_{i} \xi_{i}\left|x_{i}^{*}-y_{i}^{*}\right| & \leq \sum_{i=1}^{n} \xi_{i} \sum_{j=1}^{n} F_{j}\left(\left|b_{i j}\right|+\left|c_{i j}\right|\right)\left|x_{j}^{*}-y_{j}^{*}\right| \\
& =\sum_{i=1}^{n} \sum_{j=1}^{n} \xi_{i} F_{j}\left(\left|b_{i j}\right|+\left|c_{i j}\right|\right)\left|x_{j}^{*}-y_{j}^{*}\right| \\
& =\sum_{i=1}^{n} \sum_{j=1}^{n} \xi_{j} F_{i}\left(\left|b_{j i}\right|+\left|c_{j i}\right|\right)\left|x_{i}^{*}-y_{i}^{*}\right| \\
& =\sum_{i=1}^{n}\left[F_{i} \sum_{j=1}^{n}\left(\left|b_{j i}\right|+\left|c_{j i}\right|\right) \xi_{j}\right]\left|x_{i}^{*}-y_{i}^{*}\right| .
\end{aligned}
$$

So, we get

$$
\sum_{i=1}^{n}\left(a_{i} \xi_{i}-F_{i} \sum_{j=1}^{n}\left(\left|b_{j i}\right|+\left|c_{j i}\right|\right) \xi_{j}\right)\left|x_{i}^{*}-y_{i}^{*}\right| \leq 0 .
$$

From the assumption ( $\mathrm{H} 0)$, we get $x_{i}^{*}=y_{i}^{*}, i=1,2, \ldots, n$. Therefore, system (1) has one unique equilibrium point. The proof is complete.

\section{Global Exponential Stability of the Equilibrium Point}

In this section, we consider the following DCNN system with impulses of the type

$$
\begin{gathered}
x_{i}^{\Delta}(t)=-a_{i} x_{i}(t)+\sum_{j=1}^{n} b_{i j} f_{j}\left(x_{j}(t)\right) \\
\quad+\sum_{j=1}^{n} c_{i j} f_{j}\left(x_{j}\left(t-\tau_{i j}\right)\right)+I_{i}, \quad t \in \mathbb{T}_{0}^{+}, t \neq t_{k} \\
\Delta x_{i}\left(t_{k}\right)=x_{i}\left(t_{k}^{+}\right)-x_{i}\left(t_{k}^{-}\right)=P_{i}\left(x_{i}\left(t_{k}\right)\right) \\
=-\gamma_{i k}\left(x_{i}\left(t_{k}\right)-x_{i}^{*}\right) \\
k=1,2, \ldots, i=1,2, \ldots, n
\end{gathered}
$$

where $a_{i}, b_{i j}, c_{i j}, I_{i}$, and $f_{j}(i, j=1,2, \ldots, n)$ are defined as those in (1) and $\tau_{i j}(i, j=1,2, \ldots, n)$ are positive constants which satisfy $t-\tau_{i j} \in \mathbb{T}$ for all $t \in \mathbb{T}, i, j=1,2, \ldots, n$. Let $\tau=\max _{1 \leq i, j \leq n}\left(\tau_{i j}\right)$. Then, the initial conditions associated with (33) are of the form

$$
x_{i}(s)=\phi_{i}(s), \quad s \in[-\tau, 0] \cap \mathbb{T},
$$

where $\phi_{i} \in C_{\mathrm{rd}}([-\tau, 0] \cap \mathbb{T}, \mathbb{R}), i=1,2, \ldots, n$ are rd-continuous.

Definition 16. Let $x^{*}=\left(x_{1}^{*}, x_{2}^{*}, \ldots, x_{n}^{*}\right)^{T}$ be an equilibrium point of (33) with initial value $\phi^{*}=\left(\varphi_{1}^{*}, \varphi_{2}^{*}, \ldots, \varphi_{n}^{*}\right)^{T}$. If there exists a positive constant $\lambda$ with $-\lambda \in \mathscr{R}^{+}$such that for $t_{0} \in[-\tau, 0]_{\mathbb{T}}$, there exists $M>1$ such that for an arbitrary solution $x(t)=\left(x_{1}(t), x_{2}(t), \ldots, x_{n}(t)\right)^{T}$ of (33) with initial value $\phi(s)=\left(\phi_{1}(s), \phi_{2}(s), \ldots, \phi_{n}(s)\right)^{T}$ satisfies

$$
\begin{array}{r}
\left|x(t)-x^{*}\right|_{1} \leq M\left\|\phi-\phi^{*}\right\| e_{-\lambda}\left(t, t_{0}\right), \\
t \in[-\tau, \infty)_{\mathbb{T}}, t \geq t_{0},
\end{array}
$$

where $\left|x(t)-x^{*}\right|_{1}=\max _{1 \leq i \leq n}\left\{\left|x_{i}(t)-x_{i}^{*}\right|\right\},\left\|\phi-\phi^{*}\right\|=$ $\max _{1 \leq i \leq n} \sup _{s \in[-\tau, 0]_{\pi}}\left\{\left|\varphi_{i}(s)-\varphi_{i}^{*}\right|\right\}$. Then the equilibrium point $x^{*}$ is said to be exponentially stable.

Now, we study the global exponential stability of the unique equilibrium to (33) on time scales by using Lyapunov method. We have the following.

Theorem 17. Let (H2) and (H) hold. Suppose further that

$$
\left(\mathrm{H}_{3}\right) 0<\gamma_{i k}<2, i=1,2, \ldots, n, k=1,2, \ldots
$$


Then, the equilibrium $x^{*}=\left(x_{1}^{*}, x_{2}^{*}, \ldots, x_{n}^{*}\right)^{T}$ of system (33) is globally exponentially stable.

Remark 18. We denote the $\mathbb{T}$-interval $[a, b]_{\mathbb{T}}$ as $[a, b]_{\mathbb{T}}:=\{t \in$ $\mathbb{T} \mid a \leq t \leq b\}$.

Now, we prove Theorem 17.

Proof. Let $y_{i}(t)=x_{i}(t)-x_{i}^{*}(i=1,2, \ldots, n)$. Then, we can rewrite (33) as

$$
\begin{gathered}
y_{i}^{\Delta}(t)=-a_{i} y_{i}(t)+\sum_{j=1}^{n} b_{i j}\left[f_{j}\left(y_{j}(t)+x_{j}^{*}\right)-f_{j}\left(x_{j}^{*}\right)\right] \\
+\sum_{j=1}^{n} c_{i j}\left[f_{j}\left(y_{j}\left(t-\tau_{i j}\right)+x_{j}^{*}\right)-f_{j}\left(x_{j}^{*}\right)\right], \\
t \neq t_{k}, t \in \mathbb{T}_{0}^{+}, \\
y_{i}\left(t_{k}^{+}\right)=y_{i}\left(t_{k}\right)-\gamma_{i k} y_{i}\left(t_{k}\right), \quad i=1,2, \ldots, n, k=1,2, \ldots
\end{gathered}
$$

Multiplying both sides of the first equation of (4.2) by $e_{-a_{i}}(t, \sigma(s))$ and integrating on $\left[t_{0}, t\right]_{\mathbb{T}}$, where $t_{0} \in[-\tau, 0]_{\mathbb{T}}$, we get

$$
\begin{aligned}
& y_{i}(t)=y_{i}\left(t_{0}\right) e_{-a_{i}}\left(t, t_{0}\right) \\
& +\int_{t_{0}}^{t} e_{-a_{i}}(t, \sigma(s)) \\
& \quad \times\left\{\sum_{j=1}^{n} b_{i j}\left[f_{j}\left(y_{j}(s)+x_{j}^{*}\right)-f_{j}\left(x_{j}^{*}\right)\right]\right. \\
& +\sum_{j=1}^{n} c_{i j}\left[f_{j}\left(y_{j}\left(s-\tau_{i j}\right)+x_{j}^{*}\right)\right. \\
& \left.\left.\quad-f_{j}\left(x_{j}^{*}\right)\right]\right\} \Delta s, \quad i=1,2, \ldots, n .
\end{aligned}
$$

For positive constant $\alpha<\min _{1 \leq i \leq n} a_{i}$ with $-\alpha \in \mathscr{R}^{+}$, we have $e_{\ominus \alpha}\left(t, t_{0}\right)>1$, where $t \in\left[-\tau, t_{0}\right]_{\mathbb{T}}$. Take

$$
M>\max _{1 \leq i \leq n}\left\{\frac{a_{i}}{a_{i}-\sum_{j=1}^{m} F_{j}\left(\left|b_{i j}\right|+\left|c_{i j}\right|\right)}\right\} .
$$

In view of $(\mathrm{H})$, we have $M>1$. Hence, it is obvious that

$$
|y(t)|_{1} \leq M e_{\ominus \alpha}\left(t, t_{0}\right)\left\|\phi-\phi^{*}\right\|, \quad \forall t \in\left[-\tau, t_{0}\right]_{\mathbb{T}} .
$$

We claim that

$$
|y(t)|_{1} \leq M e_{\ominus \alpha}\left(t, t_{0}\right)\left\|\phi-\phi^{*}\right\|, \quad \forall t \in\left(t_{0}, t_{1}\right]_{\mathbb{T}} .
$$

To prove this claim, we show that for any $p>1$, the following inequality holds

$$
|y(t)|_{1}<p M e_{\ominus \alpha}\left(t, t_{0}\right)\left\|\phi-\phi^{*}\right\|, \quad \forall t \in\left(t_{0}, t_{1}\right]_{\mathbb{T}} .
$$

By way of contradiction, assume that (41) does not hold. Then, there exist $\rho \in\left(t_{0}, t_{1}\right]_{\mathbb{T}}$ and $i_{0} \in\{1,2, \ldots, n\}$ such that

$$
\begin{gathered}
\left|y_{i_{0}}(\rho)\right| \geq p M e_{\ominus \alpha}\left(\rho, t_{0}\right)\left\|\phi-\phi^{*}\right\|, \\
\left|y_{i_{0}}(t)\right|<p M e_{\ominus \alpha}\left(t, t_{0}\right)\left\|\phi-\phi^{*}\right\|, \quad t \in\left(t_{0}, \rho\right)_{\mathbb{T}}, \\
\left|y_{l}(t)\right|<p M e_{\ominus \alpha}\left(t, t_{0}\right)\left\|\phi-\phi^{*}\right\|, \quad \text { for } l \neq i_{0}, \\
t \in\left(t_{0}, \rho\right]_{\mathbb{T}}, l=1,2, \ldots, n .
\end{gathered}
$$

Therefore, there must be a constant $\delta_{1} \geq 1$ such that

$$
\begin{gathered}
\left|y_{i_{0}}(\rho)\right|=\delta_{1} p M e_{\ominus \alpha}\left(\rho, t_{0}\right)\left\|\phi-\phi^{*}\right\|, \\
\left|u_{i_{0}}(t)\right|<\delta_{1} p M e_{\ominus \alpha}\left(t, t_{0}\right)\left\|\phi-\phi^{*}\right\|, \quad t \in\left(t_{0}, \rho\right)_{\mathbb{T}}, \\
\left|u_{l}(t)\right|<\delta_{1} p M e_{\ominus \alpha}\left(t, t_{0}\right)\left\|\phi-\phi^{*}\right\|, \quad \text { for } l \neq i_{0}, \\
t \in\left(t_{0}, \rho\right]_{\mathbb{T}}, \quad l=1,2, \ldots, n .
\end{gathered}
$$

Note that, in view of (37), we have

$$
\begin{aligned}
\left|y_{i_{0}}(\rho)\right|=\mid & \mid y_{i_{0}}\left(t_{0}\right) e_{-a_{i_{0}}}\left(\rho, t_{0}\right) \\
& +\int_{t_{0}}^{\rho} e_{-a_{i_{0}}}(\rho, \sigma(s)) \\
& \times\left\{\sum_{j=1}^{n} b_{i_{0} j}\left[f_{j}\left(y_{j}(s)+x_{j}^{*}\right)-f_{j}\left(x_{j}^{*}\right)\right]\right. \\
& +\sum_{j=1}^{n} c_{i_{0} j}\left[f_{j}\left(y_{j}\left(s-\tau_{i j}\right)+x_{j}^{*}\right)\right. \\
\leq & e_{-a_{i_{0}}}\left(\rho, t_{0}\right)\left\|\phi-\phi^{*}\right\| \\
& +\int_{t_{0}}^{\rho} e_{-a_{i_{0}}}(\rho, \sigma(s)) \\
& \times\left(\sum_{j=1}^{m}\left|b_{i_{0} j}\right| F_{j}\left|y_{j}(s)\right|\right. \\
& +\int_{t_{0}}^{\rho} e_{-a_{i_{0}}}(\rho, \sigma(s)) \\
& \times\left(\sum_{j=1}^{m}\left|b_{i_{0} j}\right| F_{j} \delta_{1} p M e_{\ominus \alpha}\left(s, t_{0}\right)\right. \\
\leq & \left.+\sum_{-a_{i_{0}}}^{n}\left(\rho, t_{0}\right) \| \phi-\phi_{i_{0} j}\left|F_{j}\right| y_{j}\left(s-\tau_{i_{0} j}\right) \mid\right) \Delta s
\end{aligned}
$$




$$
\begin{aligned}
& =\left\|\phi-\phi^{*}\right\| e_{\ominus \alpha}\left(\rho, t_{0}\right) e_{-a_{i_{0}} \oplus \alpha}\left(\rho, t_{0}\right) \\
& +\delta_{1} p M e_{\ominus \alpha}\left(\rho, t_{0}\right)\left\|\phi-\phi^{*}\right\| \\
& \times \int_{t_{0}}^{\rho} e_{-a_{i_{0}} \oplus \alpha}(\rho, \sigma(s)) \\
& \times\left(\sum_{j=1}^{m}\left|b_{i_{0} j}\right| F_{j} e_{\ominus \alpha}(\rho, \sigma(s))\right. \\
& \left.+\sum_{j=1}^{n}\left|c_{i_{0} j}\right| F_{j} e_{\ominus \alpha}\left(\sigma(s), s-\tau_{i_{0} j}\right)\right) \Delta s \\
& \leq\left\|\phi-\phi^{*}\right\| e_{\ominus \alpha}\left(\rho, t_{0}\right) e_{-a_{i_{0}}+\alpha}\left(\rho, t_{0}\right) \\
& +\delta_{1} p M e_{\ominus \alpha}\left(\rho, t_{0}\right)\left\|\phi-\phi^{*}\right\| \\
& \times \int_{t_{0}}^{\rho} e_{-a_{i_{0}}}(\rho, \sigma(s)) \\
& \times\left(\sum_{j=1}^{m}\left|b_{i_{0} j}\right| F_{j}+\sum_{j=1}^{n}\left|c_{i_{0} j}\right| F_{j}\right) \Delta s \\
& \leq\left\|\phi-\phi^{*}\right\| e_{\ominus \alpha}\left(\rho, t_{0}\right) \\
& +\delta_{1} p M e_{\ominus \alpha}\left(\rho, t_{0}\right)\left\|\phi-\phi^{*}\right\| \frac{1}{-a_{i_{0}}} \\
& \times \int_{t_{0}}^{\rho}\left(-a_{i_{0}}\right) e_{-a_{i_{0}}}(\rho, \sigma(s)) \Delta s \\
& \times\left(\sum_{j=1}^{m}\left|b_{i_{0} j}\right| F_{j}+\sum_{j=1}^{n}\left|c_{i_{0} j}\right| F_{j}\right) \\
& =\left\|\phi-\phi^{*}\right\| e_{\ominus \alpha}\left(\rho, t_{0}\right) \\
& -\frac{1}{a_{i_{0}}} \delta_{1} p M e_{\ominus(-\alpha)}\left(\rho, t_{0}\right)\left\|\phi-\phi^{*}\right\| \\
& \times\left(e_{-a_{i_{0}}}\left(\rho, t_{0}\right)-1\right)\left(\sum_{j=1}^{m}\left|b_{i_{0} j}\right| F_{j}+\sum_{j=1}^{n}\left|c_{i_{0} j}\right| F_{j}\right) \\
& <\delta_{1} p M\left\|\phi-\phi^{*}\right\| e_{\ominus \alpha}\left(\rho, t_{0}\right) \\
& \times\left(\frac{1}{\delta_{1} p M}+\frac{1}{a_{i_{0}}} \sum_{j=1}^{m} F_{j}\left(\left|b_{i_{0} j}\right|+\left|c_{i_{0} j}\right|\right)\right) \\
& <\delta_{1} p M\left\|\phi-\phi^{*}\right\| e_{\ominus \alpha}\left(\rho, t_{0}\right) \\
& \times\left(\frac{1}{M}+\frac{1}{a_{i_{0}}} \sum_{j=1}^{m} F_{j}\left(\left|b_{i_{0} j}\right|+\left|c_{i_{0} j}\right|\right)\right) \\
& <\delta_{1} p M e_{\ominus \alpha}\left(\rho, t_{0}\right)\left\|\phi-\phi^{*}\right\| .
\end{aligned}
$$

Thus, we get a contradiction. Hence, (41) holds. Let $p \rightarrow$ 1 ; then (40) holds. From (40), we have that $\left|y_{i}\left(t_{1}\right)\right| \leq$
$M e_{\ominus \alpha}\left(t, t_{0}\right)\left\|\phi-\phi^{*}\right\|, i=1,2, \ldots, n$. Since $\left|y_{i}\left(t_{1}^{+}\right)\right|=\mid 1-$ $\gamma_{i 1}|| y_{i}\left(t_{1}\right)|<| y_{i}\left(t_{1}\right) \mid, i=1,2, \ldots, n$, it follows that

$$
\begin{array}{r}
\left|y_{i}\left(t_{1}^{+}\right)\right| \leq M e_{\ominus \alpha}\left(t, t_{0}\right)\left\|\phi-\phi^{*}\right\|, \\
\forall t \in\left[-\tau, t_{1}\right]_{\mathbb{T}}, i=1,2, \ldots, n .
\end{array}
$$

Thus, for $t \in\left[t_{1}, t_{2}\right]_{\mathbb{T}}$, we may repeat the above procedure and obtain

$$
\begin{gathered}
\left|y_{i}(t)\right| \leq M e_{\ominus \alpha}\left(t, t_{0}\right)\left\|\phi-\phi^{*}\right\|, \\
\forall t \in\left[t_{1}, t_{2}\right]_{\mathbb{T}}, \quad i=1,2, \ldots, n .
\end{gathered}
$$

Similarly, we have

$$
\begin{gathered}
\left|y_{i}(t)\right| \leq M e_{\ominus \alpha}\left(t, t_{0}\right)\left\|\phi-\phi^{*}\right\|, \\
\forall t \in[-\tau, \infty)_{\mathbb{T}}, \quad i=1,2, \ldots, n .
\end{gathered}
$$

Take $-\lambda=\ominus \alpha$; then $\lambda>0$ and $-\lambda \in \mathscr{R}^{+}$. Hence, we have that

$$
\begin{array}{r}
|y(t)|_{1} \leq M\left\|\phi-\phi^{*}\right\| e_{-\lambda}\left(t, t_{0}\right), \\
t \in[-\tau, \infty)_{\mathbb{T}}, t \geq t_{0},
\end{array}
$$

which means that the equilibrium point $x^{*}$ of (33) is exponentially stable. This completes the proof.

Remark 19. If the time scale $\mathbb{T}=\mathbb{R}$, then $\mu(t)=0$ and system (33) becomes the following model:

$$
\begin{aligned}
\frac{\mathrm{d} x_{i}(t)}{\mathrm{d} t}= & -a_{i} x_{i}(t)+\sum_{j=1}^{n} b_{i j} f_{j}\left(x_{j}(t)\right) \\
& +\sum_{j=1}^{n} c_{i j} f_{j}\left(x_{j}\left(t-\tau_{i j}\right)\right)+I_{i}, \quad t \neq t_{k}, \\
x_{i}\left(t_{k}^{+}\right)= & x_{i}\left(t_{k}\right)-\gamma_{i k}\left(x_{i}\left(t_{k}\right)-x_{i}^{*}\right), \\
& k=1,2, \ldots, i=1,2, \ldots, n .
\end{aligned}
$$

From Theorem 17, we can immediately derive the following result.

Corollary 20. Suppose that system (49) satisfies condition (H2) and (H), and the following assumptions hold:
(1) $-a_{i}+\sum_{j=1}^{n} F_{j}\left(\left|b_{i j}\right|+\left|c_{i j}\right|\right)<0$;
(2) $0<\gamma_{i k}<2$.

Then, the equilibrium of system (49) is globally exponentially stable.

Remark 21. In [42], by utilizing the time scale calculus theory, topological degree theory, and Hölder's inequality on time scales, authors studied the existence and the global exponential stability of equilibrium point to a class of impulsive BAM neural networks with distributed delays on time scales. But, results obtained in [42] cannot be applied to (1). Also, for establishing the global exponential stability of equilibrium point to (1), our method used in this paper is totally different from that used in [42]. 


\section{An Example}

In this section, an example is given to show the effectiveness of the result obtained in the previous section. Because the condition (4.2) is not dependent on the impulses, we just need to check it with the nonimpulsive system.

Consider the following simple DCNN on time scale $\mathbb{T}$ :

$$
\begin{aligned}
x_{i}^{\Delta}(t)= & -a_{i} x_{i}(t)+\sum_{j=1}^{n} b_{i j} f_{j}\left(x_{j}(t)\right) \\
& +\sum_{j=1}^{n} c_{i j} f_{j}\left(x_{j}\left(t-\tau_{i j}\right)\right)+I_{i}, \quad t \in \mathbb{T}_{0}^{+},
\end{aligned}
$$

where $\left(a_{1}, a_{2}\right)^{T}=(1,1)^{T}, I_{i}=2, \tau_{i j}=2(i, j=1,2)$,

$$
\left(\begin{array}{ll}
b_{11} & b_{12} \\
b_{21} & b_{22}
\end{array}\right)=\left(\begin{array}{ll}
0.01 & 0.01 \\
0.01 & 0.01
\end{array}\right), \quad\left(\begin{array}{ll}
c_{11} & c_{12} \\
c_{21} & c_{22}
\end{array}\right)=\left(\begin{array}{ll}
0.02 & 0.03 \\
0.03 & 0.02
\end{array}\right) .
$$

Taking $f_{1}(x)=f_{2}(x)=(1 / 2)(|x+1|+|x-1|)$, we can easily see that $F_{1}=F_{2}=1$.

Let $\mathbb{T}=\bigcup_{k=0}^{\infty}[2 k, 2 k+1]$, then for $t \in \mathbb{T}, t \neq 2 k+1(k=$ $0,1,2, \ldots)$, we have $\mu(t)=0$, and for $t=2 k+1(k=$ $0,1,2, \ldots)$, we have $\mu(t)=1$.

We have that

$$
\begin{aligned}
& a_{1}-\sum_{j=1}^{2} F_{j}\left(\left|b_{1 j}\right|+\left|c_{1 j}\right|\right)=0.93>0, \\
& a_{2}-\sum_{j=1}^{2} F_{j}\left(\left|b_{2 j}\right|+\left|c_{2 j}\right|\right)=0.93>0,
\end{aligned}
$$

which imply that the assumption $(\mathrm{H})$ of Theorem 14 holds. Thus, it follows from Theorems 14 and 17 that system (50) has a unique equilibrium point which is globally exponentially stable (see Figure 1).

Since $\mu(t) \equiv 0$ for $t \in \mathbb{T}=\mathbb{R}$ and $\mu(t) \equiv 1$ for $t \epsilon$ $\mathbb{T}=\mathbb{Z}$, from the discussion above one can easily see that for $\mathbb{T}=\mathbb{R}$ or $\mathbb{T}=\mathbb{Z},(50)$ always has a unique equilibrium point which is globally exponentially stable. That is, the following continuous-time system

$$
\begin{aligned}
x_{i}^{\prime}(t)= & -a_{i} x_{i}(t)+\sum_{j=1}^{n} b_{i j} f_{j}\left(x_{j}(t)\right) \\
& +\sum_{j=1}^{n} c_{i j} f_{j}\left(x_{j}\left(t-\tau_{i j}\right)\right)+I_{i}, \quad t \in \mathbb{R}^{+}
\end{aligned}
$$

and its discrete-time analogue

$$
\begin{aligned}
\Delta x_{i}(t)= & -a_{i} x_{i}(t)+\sum_{j=1}^{n} b_{i j} f_{j}\left(x_{j}(t)\right) \\
& +\sum_{j=1}^{n} c_{i j} f_{j}\left(x_{j}\left(t-\tau_{i j}\right)\right)+I_{i}, \quad t \in \mathbb{Z}^{+}
\end{aligned}
$$

have the same dynamical properties, where $a_{i}, I_{i}, \tau_{i j}$, and $f_{j}$ are the same as those in (50) (see Figures 2 and 3 ).
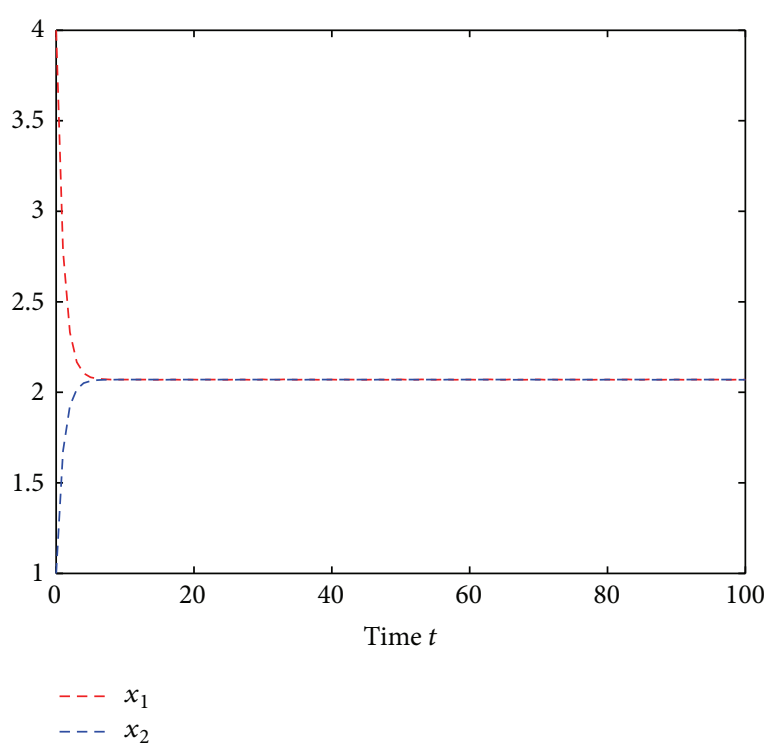

FIGURE 1: Transient responses of states $x_{1}, x_{2}$ in Example when $\mathbb{T}=$ $\bigcup_{k=1}^{\infty}[2 k, 2 k+1]$.

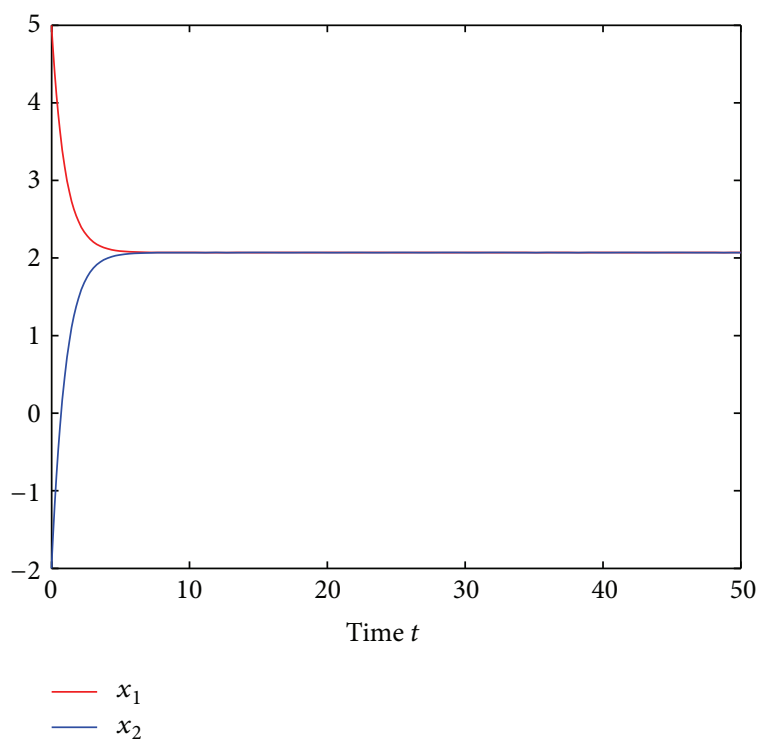

FIGURE 2: Transient responses of states $x_{1}, x_{2}$ in Example when $\mathbb{T}=$ $\mathbb{R}$.

\section{Conclusion}

Using the topological degree theory and the time scale calculus theory, some sufficient conditions are obtained to ensure the existence and the global exponential stability of equilibria for DCNNs neural networks with impulses on time scales. This is the first time to apply the time scale calculus theory to unify the study of the stability of the equilibrium for DCNNs with impulses on time scales under the same framework. The results obtained in this paper possess highly important significance and are easily checked in practice. In addition, the method in this paper may be applied to 


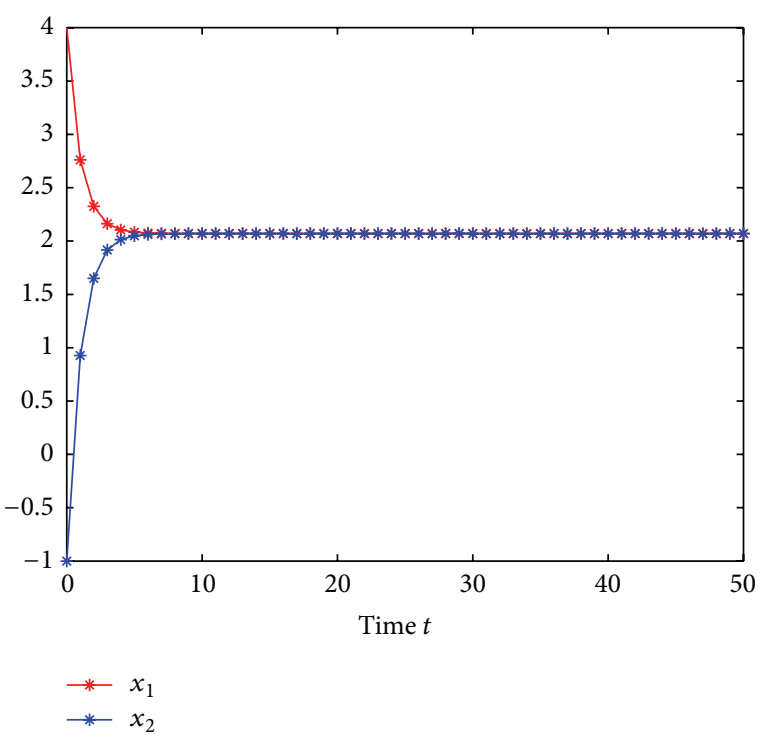

FIGURE 3: Transient responses of states $x_{1}, x_{2}$ in Example when $\mathbb{T}=$ $\mathbb{Z}$.

some other systems such as the BAM and Cohen-Grossberg systems with impulses and so on.

\section{Conflict of Interests}

The authors declare that there is no conflict of interests regarding the publication of this paper.

\section{Acknowledgments}

This work is supported by the National Natural Sciences Foundation of People's Republic of China under Grant no. 11361072 and the YNU Postdoctoral Science Foundation Project under Grant no. W4030002.

\section{References}

[1] L. O. Chua and L. Yang, "Cellular neural networks: theory," IEEE Transactions on Circuits and Systems, vol. 35, no. 10, pp. 12571272, 1988.

[2] L. O. Chua and L. Yang, "Cellular neural networks: applications," IEEE Transactions on Circuits and Systems, vol. 35, no. 10, pp. 1273-1290, 1988.

[3] L. O. Chua and T. Roska, "Stability of a class of nonreciprocal cellular neural networks," IEEE Transactions on Circuits and Systems I, vol. 37, no. 12, pp. 1520-1527, 1990.

[4] J. Cao, "A set of stability criteria for delayed cellular neural networks," IEEE Transactions on Circuits and Systems I, vol. 48, no. 4, pp. 494-498, 2001.

[5] J. Cao, "On stability of delayed cellular neural networks," Physics Letters A, vol. 261, no. 5-6, pp. 303-308, 1999.

[6] J. Cao, "On stability of cellualr neural networks with delay," IEEE Transactions on Circuits and Systems I, vol. 40, no. 3, pp. 157-165, 1993.
[7] S. Arik and V. Tavsanoglu, "On the global asymptotic stability of delayed cellular neural networks," IEEE Transactions on Circuits and Systems I, vol. 47, no. 4, pp. 571-574, 2000.

[8] H. Huang and J. Cao, "On global asymptotic stability of recurrent neural networks with time-varying delays," Applied Mathematics and Computation, vol. 142, no. 1, pp. 143-154, 2003.

[9] J. H. Park, "A new stability analysis of delayed cellular neural networks," Applied Mathematics and Computation, vol. 181, no. 1, pp. 200-205, 2006.

[10] B. Wu, Y. Liu, and J. Lu, "New results on global exponential stability for impulsive cellular neural networks with any bounded time-varying delays," Mathematical and Computer Modelling, vol. 55, no. 3-4, pp. 837-843, 2012.

[11] X. Song, X. Xin, and W. Huang, "Exponential stability of delayed and impulsive cellular neural networks with partially Lipschitz continuous activation functions," Neural Networks, vol. 29-30, pp. 80-90, 2012.

[12] J. Tian, S. Zhong, and Y. Wang, "Improved exponential stability criteria for neural networks with time-varying delays," Neurocomputing, vol. 97, pp. 164-173, 2012.

[13] S. Long and $\mathrm{D}$. $\mathrm{Xu}$, "Global exponential stability of nonautonomous cellular neural networks with impulses and timevarying delays," Communications in Nonlinear Science and Numerical Simulation, vol. 18, no. 6, pp. 1463-1472, 2013.

[14] V. Lakshmikantham, D. D. Bainov, and P. S. Simeonov, Theory of Impulsive Differential Equations, vol. 6, World Scientific, Singapore, 1989.

[15] Y. Li, "Global exponential stability of BAM neural networks with delays and impulses," Chaos, Solitons \& Fractals, vol. 24, no. 1, pp. 279-285, 2005.

[16] Y. Li and L. Lu, "Global exponential stability and existence of periodic solution of Hopfield-type neural networks with impulses," Physics Letters A, vol. 333, no. 1-2, pp. 62-71, 2004.

[17] H. Akça, R. Alassar, V. Covachev, Z. Covacheva, and E. Al-Zahrani, "Continuous-time additive Hopfield-type neural networks with impulses," Journal of Mathematical Analysis and Applications, vol. 290, no. 2, pp. 436-451, 2004.

[18] Y. Li, C. Liu, and L. Zhu, "Global exponential stability of periodic solution for shunting inhibitory CNNs with delays," Physics Letters A, vol. 337, no. 1-2, pp. 46-54, 2005.

[19] Z. Luo and J. Shen, "Stability results for impulsive functional differential equations with infinite delays," Journal of Computational and Applied Mathematics, vol. 131, no. 1-2, pp. 55-64, 2001.

[20] K. Li, "Delay-dependent stability analysis for impulsive BAM neural networks with time-varying delays," Computers \& Mathematics with Applications, vol. 56, no. 8, pp. 2088-2099, 2008.

[21] Y. Li, X. Chen, and L. Zhao, "Stability and existence of periodic solutions to delayed Cohen-Grossberg BAM neural networks with impulses on time scales," Neurocomputing, vol. 72, no. 7-9, pp. 1621-1630, 2009.

[22] Y. Li and Z. Xing, "Existence and global exponential stability of periodic solution of CNNs with impulses," Chaos, Solitons \& Fractals, vol. 33, no. 5, pp. 1686-1693, 2007.

[23] B. Lisena, "Exponential stability of Hopfield neural networks with impulses," Nonlinear Analysis: Real World Applications, vol. 12, no. 4, pp. 1923-1930, 2011.

[24] B. Aulbach and S. Hilger, "A unified approach to continuous and discrete dynamics," in Qualitative Theory of Differential Equations (Szeged, 1988), vol. 53 of Colloquium Mathematical Society János Bolyai, pp. 37-56, North-Holland, Amsterdam, The Netherlands, 1990. 
[25] B. Aulbach and S. Hilger, "Linear dynamic processes with inhomogeneous time scale," in Nonlinear Dynamics and Quantum Dynamical Systems (Gaussig, 1990), vol. 59 of Mathematical Research, pp. 9-20, Akademie, Berlin, Germany, 1990.

[26] S. Hilger, "Analysis on measure chains-a unified approach to continuous and discrete calculus," Results in Mathematics, vol. 18, no. 1-2, pp. 18-56, 1990.

[27] H. Xu, Y. Chen, and K. L. Teo, "Global exponential stability of impulsive discrete-time neural networks with time-varying delays," Applied Mathematics and Computation, vol. 217, no. 2, pp. 537-544, 2010.

[28] J. Yu, K. Zhang, and S. Fei, "Exponential stability criteria for discrete-time recurrent neural networks with time-varying delay," Nonlinear Analysis: Real World Applications, vol. 11, no. 1, pp. 207-216, 2010.

[29] Z. Liu, S. Lü, S. Zhong, and M. Ye, "Improved exponential stability criteria for discrete-time neural networks with timevarying delay," Neurocomputing, vol. 73, no. 4-6, pp. 975-985, 2010.

[30] Y. Li, "Global stability and existence of periodic solutions of discrete delayed cellular neural networks," Physics Letters A, vol. 333, no. 1-2, pp. 51-61, 2004.

[31] E. R. Kaufmann and Y. N. Raffoul, "Periodic solutions for a neutral nonlinear dynamical equation on a time scale," Journal of Mathematical Analysis and Applications, vol. 319, no. 1, pp. 315-325, 2006.

[32] R. Agarwal, M. Bohner, D. O'Regan, and A. Peterson, "Dynamic equations on time scales: a survey," Journal of Computational and Applied Mathematics, vol. 141, no. 1-2, pp. 1-26, 2002.

[33] M. Bohner and A. Peterson, Dynamic Equations on Time Scales: An Introduction with Applications, Birkhäuser, Boston, Mass, USA, 2001.

[34] M. Bohner and A. Peterson, Advances in Dynamic Equations on Time Scales, Birkhäuser, Boston, Mass, USA, 2003.

[35] Y. Xing, M. Han, and G. Zheng, "Initial value problem for first-order integro-differential equation of Volterra type on time scales," Nonlinear Analysis: Theory, Methods \& Applications, vol. 60, no. 3, pp. 429-442, 2005.

[36] Y. Li and J. Shu, "Anti-periodic solutions to impulsive shunting inhibitory cellular neural networks with distributed delays on time scales," Communications in Nonlinear Science and Numerical Simulation, vol. 16, no. 8, pp. 3326-3336, 2011.

[37] Y. Li and C. Wang, "Uniformly almost periodic functions and almost periodic solutions to dynamic equations on time scales," Abstract and Applied Analysis, vol. 2011, Article ID 341520, 22 pages, 2011.

[38] Y. Li and C. Wang, "Almost periodic solutions of shunting inhibitory cellular neural networks on time scales," Communications in Nonlinear Science and Numerical Simulation, vol. 17, no. 8, pp. 3258-3266, 2012.

[39] J. Zhou and Y. Li, "Sobolev's spaces on time scales and its applications to a class of second order Hamiltonian systems on time scales," Nonlinear Analysis: Theory, Methods \& Applications, vol. 73, no. 5, pp. 1375-1388, 2010.

[40] Y. Li and K. Zhao, "Robust stability of delayed reaction-diffusion recurrent neural networks with Dirichlet boundary conditions on time scales," Neurocomputing, vol. 74, no. 10, pp. 1632-1637, 2011.

[41] A. Berman and R. J. Plemmons, Nonnegative Matrices in the Mathematical Science, Academic Press, New York, NY, USA, 1979.
[42] Y. Li and S. Gao, "Global exponential stability for impulsive BAM neural networks with distributed delays on time scales," Neural Processing Letters, vol. 31, no. 1, pp. 65-91, 2010. 


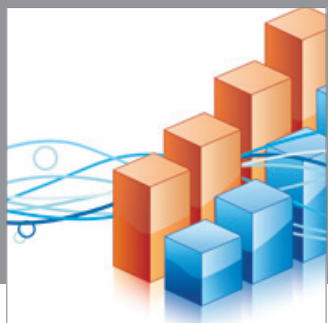

Advances in

Operations Research

mansans

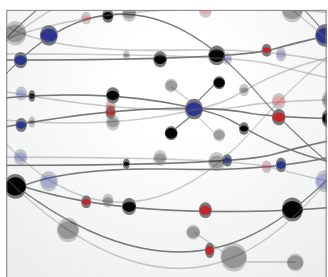

The Scientific World Journal
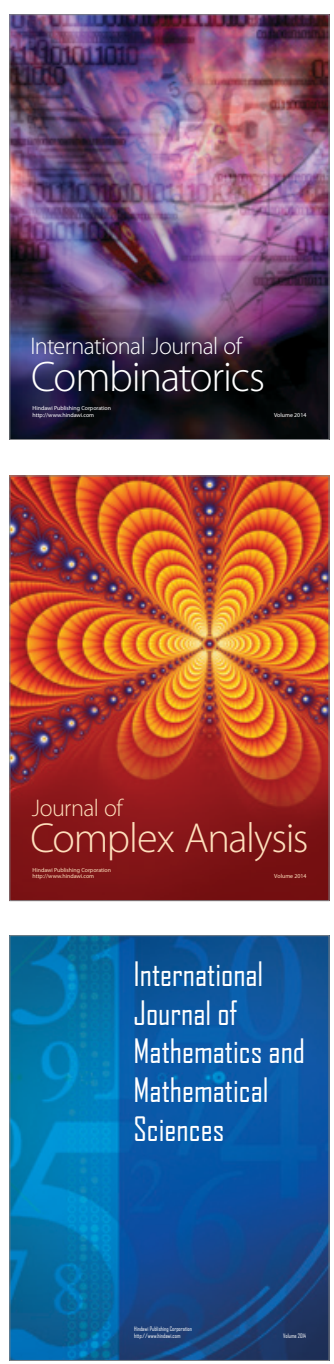
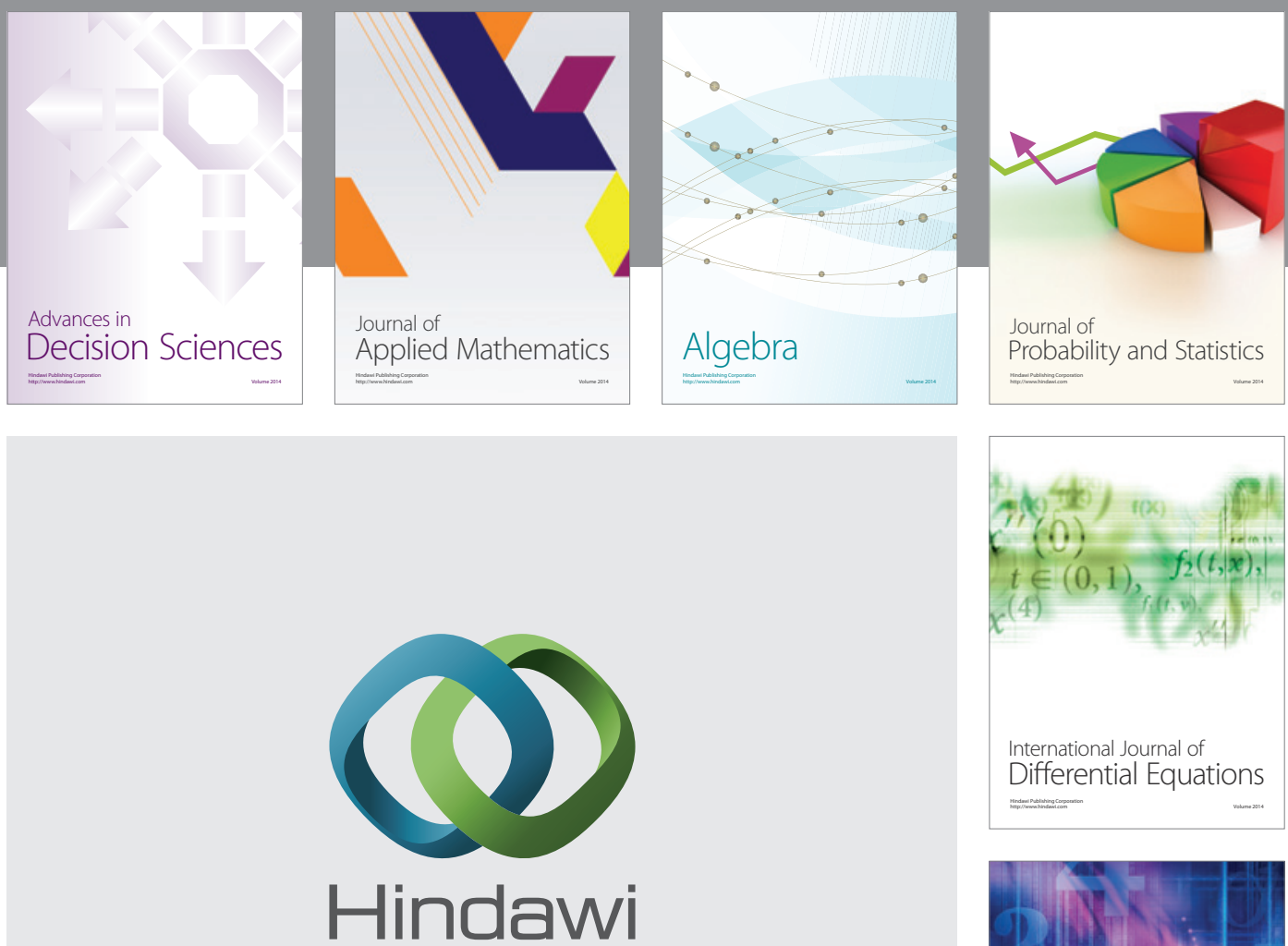

Submit your manuscripts at http://www.hindawi.com
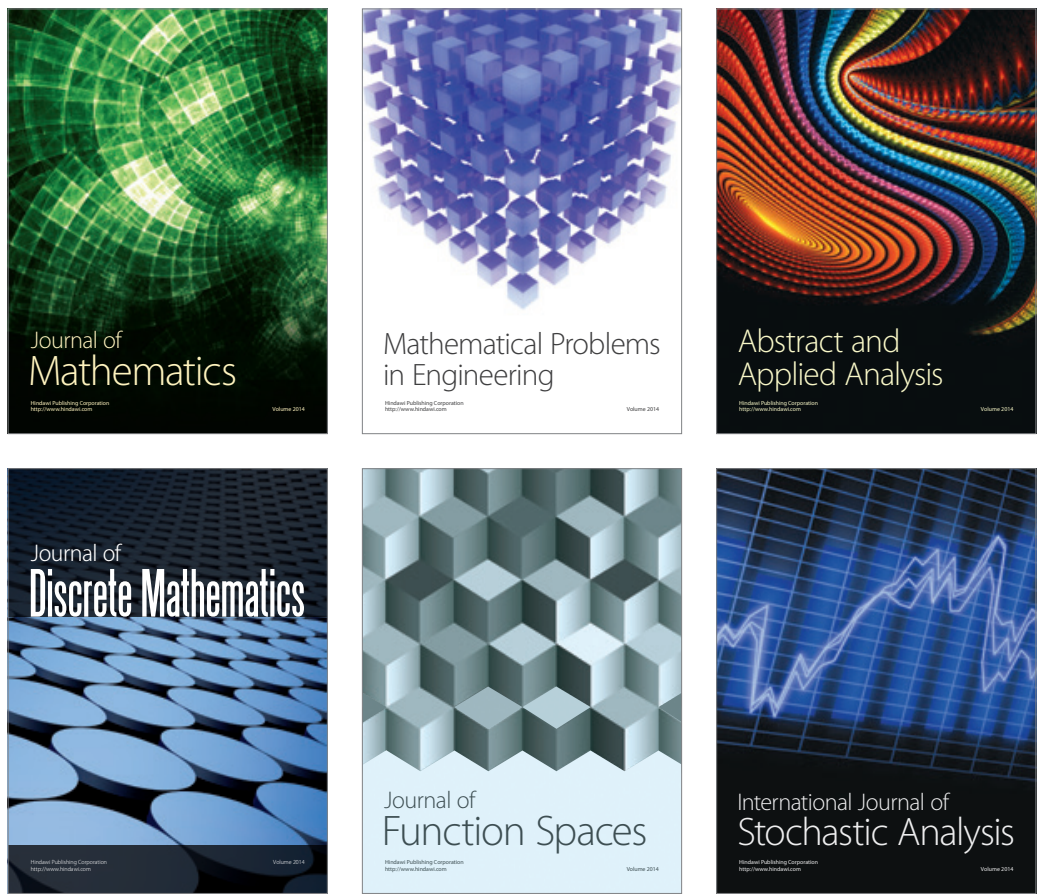

Journal of

Function Spaces

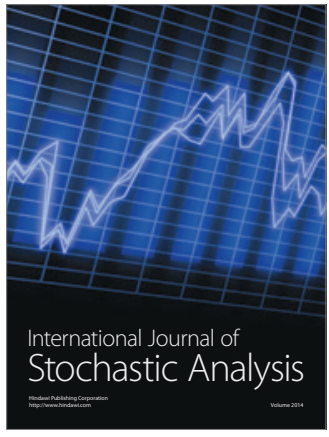

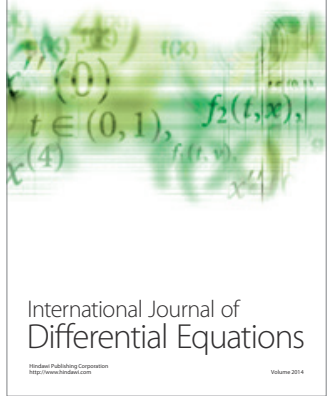
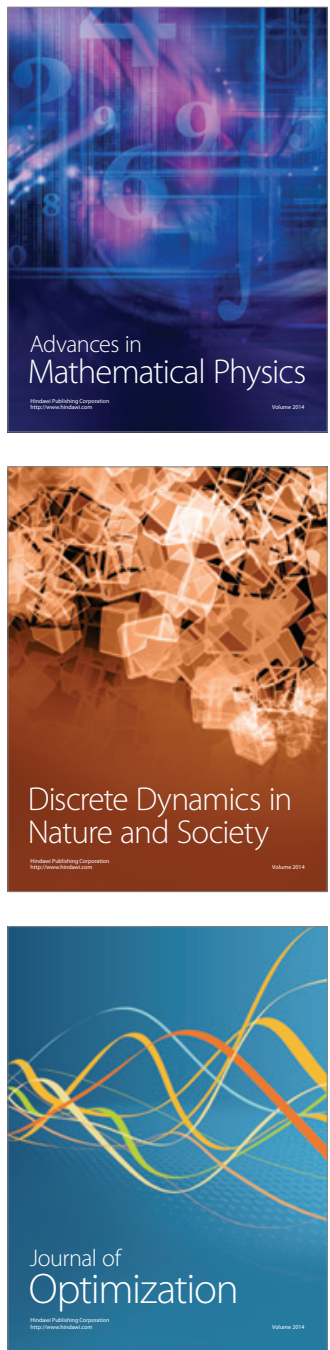